\title{
The lattice structure of mercury: Influence of electronic correlation
}

Nicola Gaston, Beate Paulus, and Krzysztof Rosciszewski*

Max-Planck-Institut für Physik komplexer Systeme,

Nöthnitzer Straße 38, D-01187 Dresden, Germany

* Institute of Physics, Jagellonian University,

Reymonta 4, Pl 30-059 Krakow, Poland

Peter Schwerdtfeger

Centre of Theoretical Chemistry and Physics, Institute of Fundamental Sciences,

Massey University (Auckland Campus), Private Bag 102904, North Shore MSC, Auckland, New Zealand

\author{
Hermann Stoll \\ Institut für Theoretische Chemie, Universität Stuttgart, \\ D-70550 Stuttgart, Germany
}

Mercury condenses at $233 \mathrm{~K}$ into the rhombohedral structure with an angle of $70.53^{\circ}$. Theoretical predictions of this structure are difficult. While a Hartree-Fock treatment yields no binding at all, density-functional (DFT) approaches with gradient-corrected functionals predict a structure with a significantly too large lattice constant and an orthorhombic angle of about $60^{\circ}$, which corresponds to an fcc structure. Surprisingly, the use of the simple LDA functional yields the correct structure and lattice constants in very good agreement with experiment; relativistic effects are shown to be essential for reaching this agreement. In addition to DFT results, we present a wavefunction-based correlation treatment of mercury and discuss in detail the effects of electron correlation on the lattice parameters of mercury including $d$-shell correlation and the influence of three-body terms in the many-body decomposition of the interatomic correlation energy. The lattice parameters obtained with this scheme at the coupled cluster level of theory, $\operatorname{CCSD}(\mathrm{T})$, agree within $1.5 \%$ with the experimental values. We further present the bulk modulus calculated within the wavefunction approach, and compare to LDA and experimental values. 


\section{INTRODUCTION}

The lattice structure of solid mercury is well known to be quite unique among metals. Adopting the rhombohedral structure, it may be described by two parameters, a bond length $a=3.005 \AA$ and rhombohedral angle $\alpha=70.53^{\circ}$ [1], cf. Fig. 1. The more common face-centred cubic (fcc) and hexagonal close packed (hcp) structures may both be related to the rhombohedral structure but in quite different ways. If one takes the rhombohedral lattice of mercury and changes $\alpha$ from $70^{\circ}$ to $60^{\circ}$, the fcc lattice is obtained with its ABCABC... layering (see Fig. 1). On the other hand, the rhombohedral lattice can be described by a hcp lattice with 3 atoms in the conventional hexagonal cell and the distance $c$ describing the repetition of three hexagonal layers (ABC). This is shown in Fig. 2. The relationship between the 3 lattices is shown in Fig. 3. Here the 12 nearest neighbours are shown such that we see the local symmetry about one central atom. While hcp has reflection symmetry through the hexagonal plane, the fcc nearest neighbors can be mapped onto one another by inversion through the centre. In this respect the rhombohedral structure is the same as the fcc structure, and therefore also has ABCABC... layering. This relationship is important to understand when examining the range of structures that deviate from the ideal hcp or fcc structures, as we will discuss.

The zinc and cadmium lattices are hcp, but known not to be ideal in that there are only 6 nearest neighbors at $a(\mathrm{nn})$ instead of 12 . These are in the hexagonal plane, while the next 6 neighbors are at a longer distance $a(\mathrm{n} 2)$ above and below the plane. Thus the distortion in zinc and cadmium corresponds to an elongation of the $c$-axis. The ratio $a(\mathrm{n} 2) / a(\mathrm{nn})$ increases going from zinc to cadmium, as seen in Tab. I, but what the connection is between this anisotropy and the anomolous structure of mercury has not been clearly explained. In the rhombohedral structure of mercury, while there are also 6 neighbors at $a(\mathrm{nn})$ and 6 neighbors at $a(\mathrm{n} 2)$, the nearest neighbors (at $a(\mathrm{nn}))$ form an octahedron instead of lying in the plane.

Currently density-functional theory (DFT) remains the method of choice for most studies involving infinite systems (for an overview see Refs. [2, 3]). Useful approximations (the local density approximation, LDA [4], and generalized gradient approximations, GGA [5]) are available and give reliable results for a wide variety of systems. Unfortunately there is no single approximation (choice of functional) that can be considered to perform best in a systematic way, just as there is no systematic way in which DFT can be improved. This is an important problem remaining, notwithstanding that DFT is in general very successful.

However DFT fails badly when an attempt is made to optimize the lattice structure of mercury. While this is known from previous work [6], we present here a systematic study of the behavior of different density functionals with respect to the lattice parameters and binding energy. Only LDA is shown to provide qualitatively and quantitatively reasonable results [6], although the agreement may be to some extent fortuitous, in view of the fact that LDA is grossly in error for small $\mathrm{Hg}$ clusters [7]. Moreover, LDA is overbinding (20-30\%) for the other group IIb metals Zn and $\mathrm{Cd}$, and cannot reproduce the experimental $c / a$ ratio (for $\mathrm{Zn}$ it is too high and for Cd too low) [8].

In previous studies $[9,10]$ we have shown that an ab initio incremental energy decomposition scheme, which corresponds to a many-body expansion of the correlation energy of the solid in terms of local entities, can accurately describe the binding of mercury as due to electron correlation. This scheme converges well with $95 \%$ of the correlation energy coming from the two-body increments and the nearest neighbor 3-body increments only. This approach has the important advantage over DFT calculations $[11,12]$ that we can reliably cover the full range from the van-derWaals bound $\mathrm{Hg}_{2}$ molecule over small and medium-size $\mathrm{Hg}$ clusters to the infinite solid. Moreover, the scheme can be systematically improved by extending the one-particle basis set, the correlation level, and the number of terms retained in the many-body expansion. Finally, we can identify different contributions to the binding of the lattice. In order to explain the adoption of the uncommon rhombohedral structure, e.g., we can analyse individual contributions from one-, two-, and three-body increments, and further identify which particular clusters in the solid are most important in fixing the lattice parameters.

The paper is organized as follows. In the next section II, the Hartree-Fock (HF) and DFT results are discussed. The influence of relativistic effects is also considered. The main part is in section III, where the correlation contributions are calculated explicitly with the method of increments, and their influence on the lattice and the bulk modulus are presented. The conclusions follow in Section IV.

\section{MEAN-FIELD RESULTS}

We performed periodic mean-field calculations for bulk $\mathrm{Hg}$ using the program package CRYSTAL [13]. In order to obtain converged results for the HF binding energy, we changed the default parameters, i.e. we set the integral threshold to $\leq 10^{-8}$ a.u. and convergence criteria for the total energy and the orbital coefficients to $10^{-6}$ a.u. and $10^{-5}$ a.u., respectively; our $k$-mesh involved $9825 k$-points in a Gilat net. The chemically inactive $[\mathrm{Kr}] 4 d^{10} 4 f^{14}$ core of the $\mathrm{Hg}$ atom was simulated by an energy-consistent scalar-relativistic pseudopotential (PP) [14]. The basis set used is a contracted Gaussian type orbital (CGTO) $(6 s 6 p 6 d) /[5 s 4 p 3 d]$ set, cf. Ref. [10]. The use of $f$ and higher polarization 
functions is currently not possible with the CRYSTAL code. For comparison, the influence of the $f$-functions can be roughly estimated from a calculation of the dimer at the solid state nearest neighbor distance, which would increase the binding only by $3 \mathrm{meV}$ per atom at the HF level. The spd part of the crystal basis set is rather compact and not optimally suited for calculating the energy of the free atom. Thus, the influence of a counterpoise correction is important when determining binding energies: its contribution is $0.217 \mathrm{eV}$ (repulsive) at the experimental structure. For determining cohesive energies and the optimum geometry we used counterpoise corrections therefore, i.e., we calculated the atomic reference energies with the crystal $((6 s 6 p 6 d) /[5 s 4 p 3 d])$ basis set at a central site and at the positions of the 12 nearest neighbors in the crystal.

We note that we neglect zero-point vibrational contributions, $E_{\mathrm{ZPVE}}$, which contribute only to about $0.01 \mathrm{eV}$ to the cohesive energy. This is much smaller than the error inherent in either DFT or the method of increments. For example, from the Debye temperature of $71.9 \mathrm{~K}$ [1] we get $E_{\mathrm{ZPVE}}=8.1 \mathrm{meV}$, and from a two-body potential [6] we obtain by extrapolation to $N \rightarrow \infty$ (of cluster sizes up to $N=40$ ) $E_{\text {ZPVE }}=8.4 \mathrm{meV}$.

Hartree-Fock calculations do not find a bound solid, nor do they bind any of the small mercury clusters [7]. Thus, binding will always be due to electronic correlation. When the distance is fixed at the experimental value, a rhombohedral angle of $90^{\circ}$ is favored. Thus HF fails completely in describing the structure of solid mercury.

At the experimental solid-state structure, the counterpoise-corrected cohesive energy (negative sign is used for attractive interactions) obtained with $\mathrm{HF}$ is $0.984 \mathrm{eV}$ (repulsive) as compared to $0.441 \mathrm{eV}$ (B3LYP), -0.091 eV (PW91), $0.054 \mathrm{eV}$ (BP86), -0.054 eV (PBE), and -0.916 eV (LDA). This compares to an experimental value of -0.67 $\mathrm{eV}$ from Ref. [1] and $-0.79 \mathrm{eV}$ from Ref. [6].

The optimized lattice parameters of solid mercury as obtained in DFT are presented in Tab. II. It is notable that the PBE, BP86, and PW91 functionals all find the fcc lattice to be the minimum structure with an angle $\alpha$ of $60^{\circ}$, while the B3LYP functional is so weakly binding that it finds a minimum at almost 90 degrees, corresponding to a simple cubic structure. LDA not only gets the rhombohedral lattice parameters correct $\left(a=2.97 \AA, \alpha=72.6^{\circ}\right)$ but also finds a much more reasonable cohesive energy $(-0.918 \mathrm{eV})$. However, this improved agreement of LDA may be partially fortuitous. Of course, one may argue that LDA which is based on the electron gas model should describe metallic systems reasonably well, but this argument does not bear out for related metals like Zn or Cd, where LDA is $20-30 \%$ overbinding and the lattice constants are correct only up to $7 \%$ [8], nor for small mercury clusters where we find a serious overbinding with LDA [7]. Nor is there a clear explanation for the wholly different performance of the other functionals. However we have shown that the rhombohedral angle increases from $60.0^{\circ}$ to $86.0^{\circ}$ as the lattice is compressed from $a=3.54$ to $a=3.00 \AA$, with PW91, [7], which implies that the correct description of the rhombohedral angle in LDA is probably related to the overbinding inherent in this functional.

We have compared the calculations described above with non-relativistic (NR) ones, using a non-relativistic pseudopotential (and corresponding basis set) [15] within the LDA. The comparison is shown in Figs. 4 and 5 for the calculation of the rhombohedral and hexagonal lattices, respectively. The most marked difference upon optimising the NR rhombohedral lattice is the change of the structure from rhombohedral to fcc, where the angle shifts to almost exactly $60^{\circ}$ at $3.10 \AA$. The NR lattice must be compressed to a bond length of $2.8 \AA$ (reducing the cohesive energy by $50 \%)$ to shift the angle $\alpha$ to $72^{\circ}$. The NR cohesive energy is slightly more binding at the minimum structure $(a=3.10$ $\left.\AA, \alpha=60.0^{\circ}, \mathrm{E}_{c o h}=-0.93 \mathrm{eV}\right)$ than the relativistic minimum, with a minimum corresponding to a bulk modulus of 0.353 Mbar. This is accidentally close to the experimental value, but a factor of two greater than the relativistic bulk modulus obtained with LDA. The enhanced lattice constant can be explained by the (unphysical) large extent of the NR $6 s$-shell. In the relativistic description, the energy minimum is much flatter and the equilibrium structure ( $\left.a=2.97 \AA, \alpha=72.6^{\circ}\right)$ is very sensitive to compression. Only slight changes to smaller lattice constants would force mercury into a simple cubic (sc) structure with an angle of $90^{\circ}$. The adoption of the rhombohedral structure can not be explained purely by the relativistic contraction of the $6 s$-shell, which is isotropic in space. The relativistic expansion of the $5 d$-shell can cause such an anisotropic distortion of the lattice.

An optimization of the NR hcp lattice for mercury shows that this is nearly ideal $(c / a=1.63)$, and about 15 meV more stable than the (almost) fcc lattice. Thus, non-relativistic mercury would be ideal hcp, according to LDA. This is in qualitative agreement with the findings of Singh [12] where only the ideal fcc and hcp lattices were considered. The relativistic hcp lattice, in contrast, turns out in LDA calculations to be even more anisotropic than Cd or Zn, but the anisotropy is inverted, leading to a much smaller $c / a$ ratio (about 1.4) than ideal. This means that hcp mercury would have weakly bound hexagonal planes compressed along the $c$-axis. In this structure the second nearest neighbor distance is $a(\mathrm{n} 2)=a_{h e x}=1.11 a(\mathrm{nn})$, slightly less than the value of $1.15 a(\mathrm{nn})$ in the rhombohedral case. This compares to $1.09 a(\mathrm{nn})$ and $1.11 a(\mathrm{nn})$ for $\mathrm{Zn}$ and $\mathrm{Cd}$, respectively, but in the case of mercury the nearest neighbor distance is not the bond length in the hexagonal plane. All these findings strongly suggest that the rhombohedral structure of mercury is of relativistic origin. 


\section{EXPLICIT TREATMENT OF ELECTRONIC CORRELATION}

\section{A. Method of increments for metals}

As described in Ref. [10], we include electronic correlation in a procedure known as the method of increments. A review of this approach describes its application to systems including van der Waals (vdW) solids, insulators and metals [16]. A general many-body expansion for the correlation energy may be written in the form

$$
E_{\mathrm{corr}}=\sum_{i} \epsilon_{i}+\sum_{i<j} \Delta \epsilon_{i j}+\sum_{i<j<k} \Delta \epsilon_{i j k}+\ldots .
$$

Such an expansion, where the $n$-body indices $i, j, k, \ldots$ number individual atoms, is known to work extremely well for vdW crystals. For noble gases $[17,18]$ it is even possible to include the HF part of the energy within the many-body expansion, and already a two-body potential (e.g. in the form of a Lennard-Jones potential) gives quite reasonable results. However, for mercury an expansion of the total energy converges only slowly [6]. This is due to the changing character of the $\mathrm{Hg}-\mathrm{Hg}$ bond in mercury, which for the dimer is indeed vdW-like, but becomes progressively more covalent as the number of atoms increases and metallic in the case of the bulk [19]. Thus the bond length in the solid is $3.00 \AA$, considerably shorter than in the dimer $(3.69 \AA)$, and already on the repulsive part of the two-body potential where higher than 2-body effects become important [10]. For the noble gases the ratio of the dimer bond length to the solid nearest neighbor distance is only 1.03, in contrast to 1.23 for mercury [18]. However, we have shown [10] that a many-body expansion of the correlation energy in embedded fragments of the solid converges much more quickly, so that the two-body part contains more than $90 \%$ of the total correlation energy. Thus even in a metal, correlation is sufficiently local to make this expansion useful.

Specifically, we use an embedding scheme where we include a shell of $\mathrm{Hg}$ atoms described with 2-valence-electron scalar relativistic pseudopotentials [20] which simulate the $\mathrm{Hg} 5 s^{2} 5 p^{6} 5 d^{10}$ shells within the atomic core, in order to model the metallic environment. Thus, only the $6 s$ shell is explicitly treated in the embedding region. Naturally, the convergence of the incremental scheme also has to be examined with respect to the size of this shell.

The critical part in the application of the method of increments to metals is the possibility of describing the metallic orbitals calculated in the embedding region in a local way. This localization is done in our approach by a unitary transformation of the occupied canonical orbitals according to the criterion of Foster and Boys [21]. Good localization characteristics are achieved by using an $s$-type atomic basis set on the embedding and thereby avoiding delocalization due to $s p$-mixing. Within this localized environment we can use the full basis for the 1,2 , or 3 atoms that we are calculating the correlation energy of, for the 1-, 2-, or 3-body increments, respectively. This approach allows us to successively include metallic delocalization in an incremental way.

The increments are therefore calculated for selected cluster models which reflect the geometry of the Hg crystal. The rhombohedral structure of the infinite crystal can be viewed as a central atom surrounded by atom shells of various size. We select for the embedding the first shell containing 12 atoms, 6 of them at distance $a(\mathrm{nn})(=3.005 \AA)$ and 6 at $1.155 a(\mathrm{nn})$; the next shell is separated by a distance of $1.12 \AA$ (for the case of the equilibrium structure). For calculating a few-body term, we include all atoms in the embedding which are in the first shell of one of the atoms to be correlated.

The basis set used on the embedding atoms is a $2 s$ basis, with contraction coefficients optimized for the free atom. The description of the atoms to be correlated is much more important for the final correlation energy, as we will keep frozen the localized orbitals of the atoms of the embedding region when calculating correlation energies. The basis sets of the correlated atoms were described in Ref. [10]. Here we use basis B as described in that paper, a $(10 s 9 p 7 d 2 f 1 g) /[8 s 7 p 6 d 2 f 1 g]$ set, and an even tempered augmented version of the same basis (aug basis B) to gain an impression of the basis set completeness for these calculations. Using these basis sets, we recalculate the integrals and reoptimize the orbitals of the atoms to be correlated, in a HF calculation, within the frozen environment of the embedding. Thus we have reasonably delocalized orbitals within the interior of our cluster over the atoms $i, j, .$. to be correlated, but localized orbitals with respect to the embedding. Then we calculate the correlation energy of these atoms in a coupled-cluster calculation with single and double excitations and perturbative treatment of the triples $(\operatorname{CCSD}(\mathrm{T}))[22,23]$. We can do this with different definitions of the core in order to see the different contributions of the valence $6 s, 5 d$, and core $5 s p$ electrons. All these calculations are performed using the MOLPRO suite of ab-initio programs [24]. 


\section{B. Results for the lattice constants}

In order to discuss the dependence of the energy of the lattice on its structure, we vary the lattice distance and angle of the structure around the experimental lattice parameters. The HF energy decreases with increasing distance (see Fig. 6) as is normal for a purely repulsive potential. It also decreases with increasing angle (see Fig. 7) which corresponds to an opening of the structure (lowering of the density), cf. Sect. II.

The one-body terms of the correlation-energy expansion are repulsive for mercury and have nearly no dependence on the lattice parameter. The first term in the expansion to examine closely with respect to the effect of the lattice parameter is therefore the two-body increment. This is shown in Fig. 6 with respect to the Hg-Hg distance. Here the potential is actually very flat, and thus the minimum can be shifted noticeably by the use of an augmented basis set, if we are only considering the two-body increments. In Fig. 7 the angular dependence of the two-body part is shown to be even weaker. Only with the augmented basis set does a shallow minimum appear. However the basis-set dependence is almost negligible with respect to the overall minimum once we consider the three-body contribution. Without the augmented basis, we find a minimum with $a=2.96 \AA$, and $\alpha=70.5^{\circ}$, which is at exactly the same $\mathrm{Hg}-\mathrm{Hg}$ distance but a slightly larger angle than in the augmented case. If we include only the correlation of the valence $s$-electrons in the two-body increments we still have no minimum, and no cohesion. Only with the inclusion of $d$-correlation in the two-body part do we find a bound solid.

It is clear from Figs. 6 and 7 that the three-body increments are most important in determining the correct curvature of the potential curve, with respect to the $\mathrm{Hg}-\mathrm{Hg}$ distance, as well as the rhombohedral angle of the solid. To analyse the influence of the individual 3-body contributions in detail, we show in Fig. 8 the geometries of the 3-body increments used in the calculation.

We have chosen to truncate the expansion of the three-body correlation after 8 increments. We concentrate on these eight geometries because they contribute about $80 \%$ to the 3 -body part of the cohesive energy. These 8 clusters all have 2 nearest or second-nearest-neighbor distances connecting the atoms. The three-body clusters range from the linear three-atom clusters (b and e) (with a fixed angle of 180 degrees) where we expect little angular dependence (of similar magnitude to the angular dependence of the two body contribution) to the compact triangle with two nn distances and one $1.15 a(\mathrm{nn})(\mathrm{c})$. The importance of each cluster for the cohesive energy depends on the weight factor which describes how often each geometry occurs in the solid. Thus clusters f) and h), which have weight factors of 12 , are much more influential than clusters a), b), and e), which have weight factors of 2 , 3, and 3, respectively. Clusters c), d) and g) have weight 6 each.

Figure 9 shows the dependence of the energy of each 3-body cluster on the lattice parameter $a$, at $\alpha=70.53^{\circ}$. Clusters d) and g) have most influence on determining the position of the minimum, while cluster h) has not only the most important contribution to the binding but also the greatest dependence on $a$. In this respect it is interesting that it is not the most compact nearest-neighbor interactions that have the most influence on the structure, but, especially, in the case of d) and h), extended clusters with strongly obtuse angles that are important. The three-body contribution from these clusters tends to shorten the bond length.

Fig. 10 shows the dependence on the angle at fixed experimental lattice constant. We note that the compact clusters a) and c) are those with the largest preference for larger angles. Of these, only c) has a cohesive contribution to the bulk. The clusters with obtuse angles, d), f), g) and h), have the largest contribution towards reducing the rhombohedral angle; in other words favoring the fcc structure. These all have binding contributions. Especially cluster $\mathrm{h}$ ) is responsible for about one third of the total three-body binding. The remaining clusters (the linear ones) have very little angle dependence.

The importance of the core (or semi-core) $d$-correlations for the 3-body correlation-energy increments of mercury is clear when comparing to the $s$-valence-only correlation contribution. For example, the $d$-correlation of the three-body increments alone is $30 \%$ of the three-body contribution, and accounts for $14 \%$ of the total cohesive energy (or $5 \%$ of the total correlation energy). In particular clusters c) and f), both compact clusters containing the rhombohedral angle, increase strongly in cohesion with the contribution of $d$-correlation. In contrast, cluster a), which sits in the hexagonal plane, gains nothing from $d$-correlation and even becomes more repulsive. This implies that the $d$-electrons stabilize the rhombohedral structure relative to hcp.

\section{The bulk modulus}

We have calculated the bulk modulus of mercury, using the results of our incremental method, as well as the LDA functional for comparison as it obtains reasonable lattice parameters for the rhombohedral lattice. The bulk modulus 
is defined in terms of the energy $E$ of the solid as

$$
B=V_{0} \frac{d^{2} E}{d V^{2}}=\frac{1}{9 V_{0}} \frac{d^{2} E}{d \delta^{2}}
$$

with volume $V$, where $V_{0}$ is the volume of the unstrained system, and $\delta$ is the distortion parameter which describes isotropic volume dilation. The more general case of the energy dependence on volume is written by means of a Taylor expansion in the distortion parameters, giving the elastic constants $c_{a b c d}$. The elastic constants are the second order constants in the Taylor expansion, and the $\delta_{a b}$ are distortion elements where the indices $a, b$ run over the Cartesian coordinates $x, y, z$. The elastic coordinates are usually given in the Voigt notation, where $x x=1, y y=2, z z=3$, $x y=y x=6, x z=z x=5$, and $y z=z y=4$. Here we restrict our interest to the case of the isotropic distortion from which we calculate the bulk modulus.

The experimental value is calculated from the elastic constants of Ref. [25] as reproduced in Ref. [26]. The rhombohedral lattice, as a trigonal system, has 6 independent elastic constants $c_{11}, c_{12}, c_{13}, c_{14}, c_{33}$, and $c_{44}$. The bulk modulus can be calculated for a trigonal system as

$$
B=\frac{\left(c_{11}+c_{12}\right) c_{33}-2 c_{13}^{2}}{c_{11}+c_{12}-4 c_{13}+2 c_{33}}
$$

which gives a value for the experimental bulk modulus of $B=0.322$ Mbar. We note that Refs. [11, 12] cite an experimental value of $0.382 \mathrm{Mbar}$, for which we have been unable to find a reference.

Our calculated values for the bulk modulus are given in Tab. III, both for the complete results of the method of increments, and for the case of truncation after two-body terms only. We also excluded the $d$-correlation of the 3 -body increments to give an $s$-only value for the bulk modulus. We obtained the bulk modulus by fitting a quadratic curve to the points calculated at $\alpha=70.53^{\circ}$, for values of $a=2.94,2.97,3.005$, and $3.03 \AA$. For the two-body energies only, an additional point at $3.06 \AA$, was included. The estimated error bounds of the two-body bulk modulus are therefore somewhat better than for the bulk modulus with three-body increments included.

The bulk modulus calculated with two-body increments only is 0.132 Mbar, considerably lower than the experimental value, and similar to the result of LDA (in the rhombohedral lattice), 0.187 Mbar. In the hcp lattice the LDA bulk modulus does not change much, with a value of $0.190 \mathrm{Mbar}$. This strong underestimation of the bulk modulus with LDA is in contrast to what would normally be expected for an overbound structure. The two-body increments with only $s$-correlation are still repulsive, as mentioned above. When only the $s$-correlation of the three-body increments is included, the bulk modulus increases to a value of 0.383 Mbar. The final result of the method of increments, with the inclusion of $d$-correlation for the three-body increments, gives $0.360 \mathrm{Mbar}$. This is rather better agreement with experiment than might be expected given the small number of points available to describe the distortion (and the simple quadratic fit).

\section{CONCLUDING REMARKS}

The lattice structure of solid mercury has been calculated using various mean-field methods as well as a wavefunction-based approach explicitly including electron correlation via an incremental scheme. Of the mean-field methods, only LDA yields the correct rhombohedral structure (albeit at the cost of moderate over-binding), while GGA approaches lead to a fcc structure and substantially too long nearest-neighbor distances (connected with serious under-binding). The LDA bulk modulus is strongly underestimated. With the wavefunction-based incremental scheme, we can reproduce the lattice constant and rhombohedral angle to within $99 \%$ of the experimental values, and obtain a cohesive energy of $-0.65 \mathrm{eV}, 97 \%$ of the experimental value, and the bulk modulus in very good agreement with experiment.

The influence of relativistic effects has been analyzed at the LDA level, by comparing results calculated with a nonrelativistic and a scalar-relativistic ECP. The rhombohedral structure is found to be unstable in the non-relativistic calculations; non-relativistic $\mathrm{Hg}$ would adopt a hcp structure.

An asset of the incremental scheme is the possibility to analyze various contributions to the correlation energy. Twobody correlation-energy contributions are essential to overcome Hartree-Fock repulsion. At the two-body level, the correct rhombohedral structure is already adopted, but the potentials are too flat and only 50\% of the experimental cohesive energy is recovered. For good agreement with experiment (also with respect to the bulk modulus), threebody terms in the correlation-energy expansion are required. It is important to note that correlation of the $6 s$ shell alone would not have been sufficient to reach this agreement. Without correlation contributions of the outer-core $5 d$ shell, $\mathrm{Hg}$ would still not be bound at the two-body level, and about one third of the three-body contribution to the cohesive energy would be missing. As the $d$-orbitals are considerably more anisotropic than the valence $s$-orbitals, 
their increased importance contributes to the dominance of the 3-body forces. The selection of the rhombohedral angle is seen in the $d$-correlation energy which is repulsive within the hexagonal plane, but strongly cohesive within the triangles containing the rhombohedral angle which make up the nearest neighbor octahedron.

Thus it is the close proximity of the $d$-orbitals, caused both by the strong relativistic contraction of the $\mathrm{Hg}-\mathrm{Hg}$ distance relative to the dimer, and the relativistic expansion of the $5 d$-orbitals, which combine to make the unusual structure of bulk Hg.

\section{Acknowledgements}

Financial support by the Marsden Fund administered by the Royal Society of New Zealand (Wellington) is gratefully acknowledged.

[1] CRC Handbook of Chemistry and Physics, CRC Press, New York (1997).

[2] R.M. Dreizler and E.K.U. Gross, Density Functional Theory, Springer-Verlag, Berlin (1990)

[3] C. Fiolhais, F. Nogueira, and M. Marques (eds.), A Primer in Density Functional Theory, Lecture Notes in Physics, vol. 620, Springer, Berlin, 2003.

[4] R. O. Jones and O. Gunnarsson, Rev. Mod. Phys. 61, 689 (1989).

[5] J. P. Perdew, in Density Functional Theory, edited by E. K. U. Gross and R. M. Dreizler (Plenum Press, New York, 1995), p. 51.

[6] G.E. Moyano, R. Wesendrup, T. Söhnel and P. Schwerdtfeger, Phys. Rev. Lett. 89, 103401 (2002).

[7] N. Gaston and P. Schwerdtfeger, Phys. Rev. B, in press (2006).

[8] B. Paulus, K. Rosciszewski, P. Sony and U. Wedig, in preparation.

[9] B. Paulus and K. Rosciszewski, Chem. Phys. Lett., 394, 96-100 (2004).

[10] B. Paulus, K. Rosciszewski, N. Gaston, P. Schwerdtfeger and H. Stoll, Phys. Rev. B 70, 165106 (2004).

[11] P. P. Singh, Phys. Rev. B 49, 4954-4958 (1994).

[12] P. P. Singh, Phys. Rev. Lett. 72, 2446-2450 (1994).

[13] V.R. Saunders, R. Dovesi, C. Roetti, et. al. program Crystal 98, Theoretical Chemistry Group, University of Torino (1998).

[14] D. Andrae, U. Häussermann, M. Dolg, H. Stoll, and H. Preuss, Theor. Chim. Acta 77, 123 (1990). (The $f$ and $g$ projectors are omitted in the Crystal code.)

[15] U. Haeussermann, (1988). Available from www.theochem.uni-stuttgart.de/pseudopotentials/

[16] B. Paulus, Phys. Rep. 421, 1 (2006).

[17] K. Rosciszewski, B. Paulus, P. Fulde and H. Stoll, Phys. Rev. B 60, 7905 (1999).

[18] P. Schwerdtfeger, N. Gaston, R. P. Krawczyk, R. Tonner, and G.E. Moyano, Phys. Rev. B 73, 064112 (2006).

[19] H. Haberland, H. Kornmeier, H. Langosch, M. Oschwald and G. Tanner, J. Chem. Soc. Faraday Trans. 86, 2473 (1990).

[20] W. Küchle, M. Dolg, H. Stoll, and H. Preuss, Mol. Phys. 74, 1245 (1991).

[21] J.M. Foster and S.F. Boys, Rev. Mod. Phys. 32, 296 (1960).

[22] C. Hampel, K. Peterson, and H.-J. Werner, Chem. Phys. Lett. 190 (1992) 1.

[23] M. J. O. Deegan and P. J. Knowles, Chem. Phys. Lett 227 (1994) 321.

[24] MOLPRO version 2002.6 - a package of ab-initio programs written by H.-J. Werner and P. J. Knowles and R. Lindh and M. Schütz and others, (see http://www.molpro.net), Birmingham, UK, 2003.

[25] E. Grüneisen and O. Sckell, Ann. Physik 19, 387 (1934).

[26] H. B. Huntingdon, Solid State Phys. 7, 282 (1958). 
Structure $a(\mathrm{n} 2) / a(\mathrm{nn})$ layering $\quad \alpha$

\begin{tabular}{rrr}
\hline \hline & & \\
hcp (ideal) & 1.000 & ABA 60.00 \\
beryllium (hcp) & 0.974 & ABA 59.10 \\
magnesium (hcp) & 0.996 & ABA 59.87 \\
zinc (hcp) & 1.093 & ABA 62.78 \\
cadmium (hcp) & 1.106 & ABA 63.12 \\
mercury (rhomb) & 1.153 & ABC 70.53 \\
simple cubic & 1.414 & -90.00 \\
fcc (ideal) & 1.000 & ABC 60.00 \\
\hline
\end{tabular}

TABLE I: Different structures of simple metals with the corresponding $a(n 2) / a(n n)$ ratio. hcp=hexagonal close-packed, fcc $=$ face-centred cubic, and rhomb=rhombohedral. The layering pattern between hexagonal planes is given. $\alpha$ corresponds to the angle between in plane and out of plane bonds in the hcp structure, and the angle between two bonds $a(\mathrm{nn})$ in the rhombohedral structure.

Functional $a \quad \alpha \quad \mathrm{E}_{\text {coh }} \quad a(\mathrm{n} 2) / a(\mathrm{nn})$

\begin{tabular}{lllll}
\hline \hline & & & & \\
B3LYP & 3.894 & 89.5 & -0.044 & 1.408 \\
PW91 & 3.535 & 61.2 & -0.195 & 1.015 \\
BP86 & 3.539 & 63.7 & -0.078 & 1.044 \\
PBE & 3.540 & 60.9 & -0.164 & 1.014 \\
LDA & 2.971 & 72.6 & -0.918 & 1.182 \\
& & & & \\
\hline & & & & \\
Expt & 3.005 & 70.53 & -0.67 & 1.153 \\
\end{tabular}

TABLE II: DFT-results for lattice structure of solid mercury. Calculated lattice parameters are in $\AA$ and cohesive energies are in $\mathrm{eV}$. (We use negative values throughout as not all our calculated values (e.g. in Hartree-Fock) are binding). The experimental values are from Ref. [1]. 


\begin{tabular}{llllll} 
Method & $a$ & $\alpha$ & $\mathrm{E}_{\text {coh }}$ & $\mathrm{B}$ \\
& & & & \\
\hline \hline & & & & & \\
NR-LDA & 3.10 & 60.0 & -0.930 & 0.353 \\
LDA & 2.97 & 72.6 & -0.918 & 0.187 \\
LDA (hcp) & 3.06 & 60.0 & -0.813 & 0.190 \\
Incremental correlation (2b:aug. basis) & 2.97 & 70.0 & -0.375 & 0.132 \\
Incremental correlation (3b:s-only) & 2.97 & 69.2 & -0.561 & 0.383 \\
Incremental correlation (3b) & 2.96 & 69.5 & -0.649 & 0.360 \\
Expt [1] & 3.005 & 70.53 & -0.670 \\
(LMTO):fcc [12] & 5.03 & 60.0 & & 0.48 \\
(LMTO):hcp [12] & 3.58 & 60.0 & & 0.21 \\
Expt [12] & & & & & 0.382 \\
Expt [26] & & & & 0.322
\end{tabular}

TABLE III: Results for the structure and bulk modulus of solid mercury. $a$ is in $\AA$, $\alpha$ in degrees, $\mathrm{E}_{\text {coh }}$ in $\mathrm{eV}$ and $\mathrm{B}$ in Mbar. For the LDA value in the hcp structure, $a=a(\mathrm{nn})$ is given.

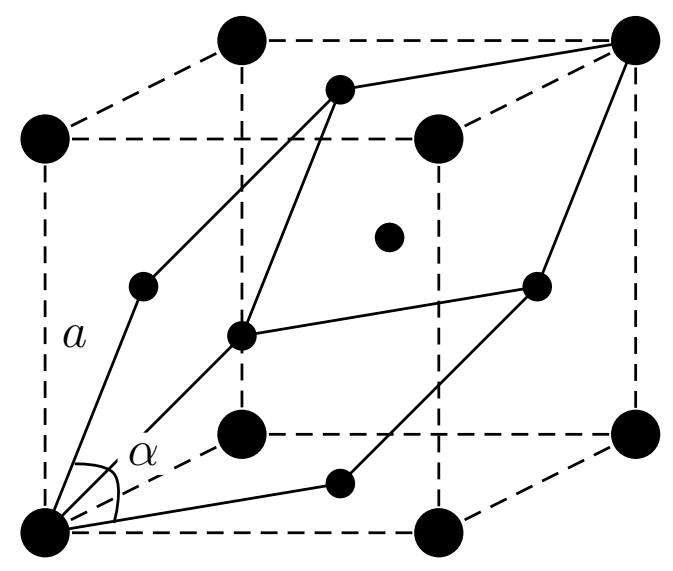

FIG. 1: The rhombohedral primitive cell is shown with reference to the face-centered cubic (fcc) structure. If the angle $\alpha$ is $60^{\circ}$, it is an ideal fcc close packed structure. This angle is $70^{\circ}$ in $\mathrm{Hg}$. If this angle were $90^{\circ}$, then it would be a simple cubic lattice. An angle of $109.28^{\circ}$ corresponds to a body-centered cubic (bcc) lattice. 


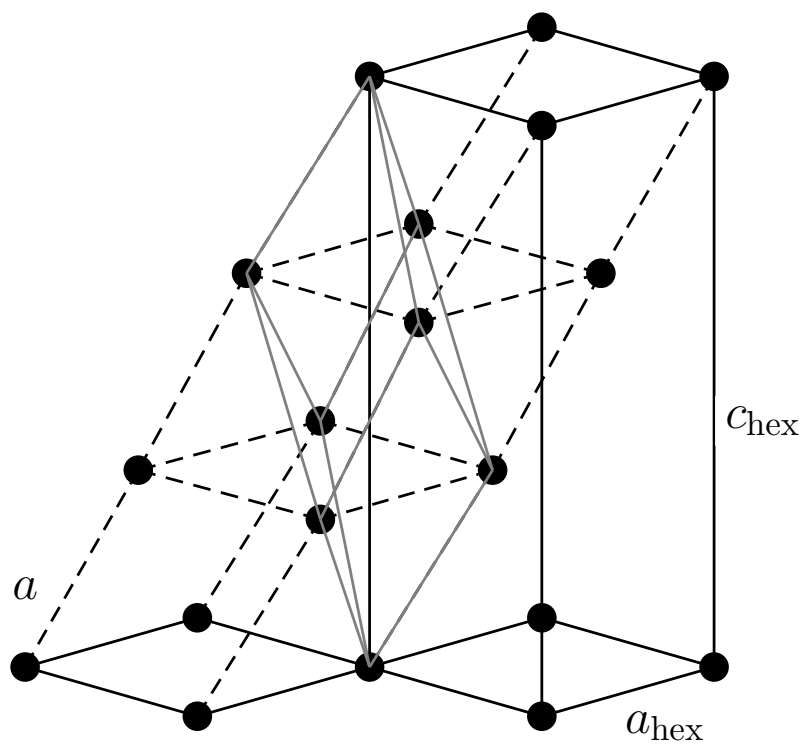

FIG. 2: The rhombohedral primitive cell is shown with reference to the hexagonal close-packed (hcp) structure, which is described by two lattice parameters (here labelled $a_{\text {hex }}$ and $c_{\text {hex }}$ ). The primitive cell in the hexagonal cell is also shown (grey lines). The conventional cell in the hcp lattice contains 3 atoms.
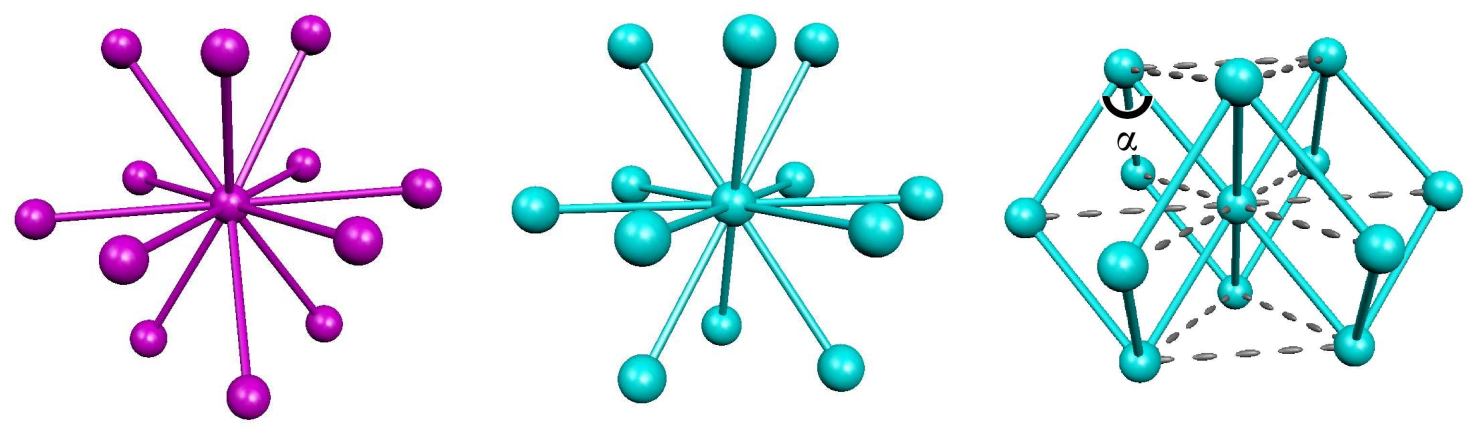

FIG. 3: The relationship of the hcp (left), fcc (middle), and rhombohedral (right) lattices is shown in terms of the arrangement of the 12 nearest neighbours in the solid. For the rhombohedral case, the rhombohedral lattice parameter, $a=a(n n)$, is shown with solid bonds while the second nearest neighbour $a(n 2)$ is shown with dotted bonds. It should be possible to see that the stacking order in the rhombohedral lattice is $\mathrm{ABC}$ as in fcc, as opposed to ABA in hcp. 


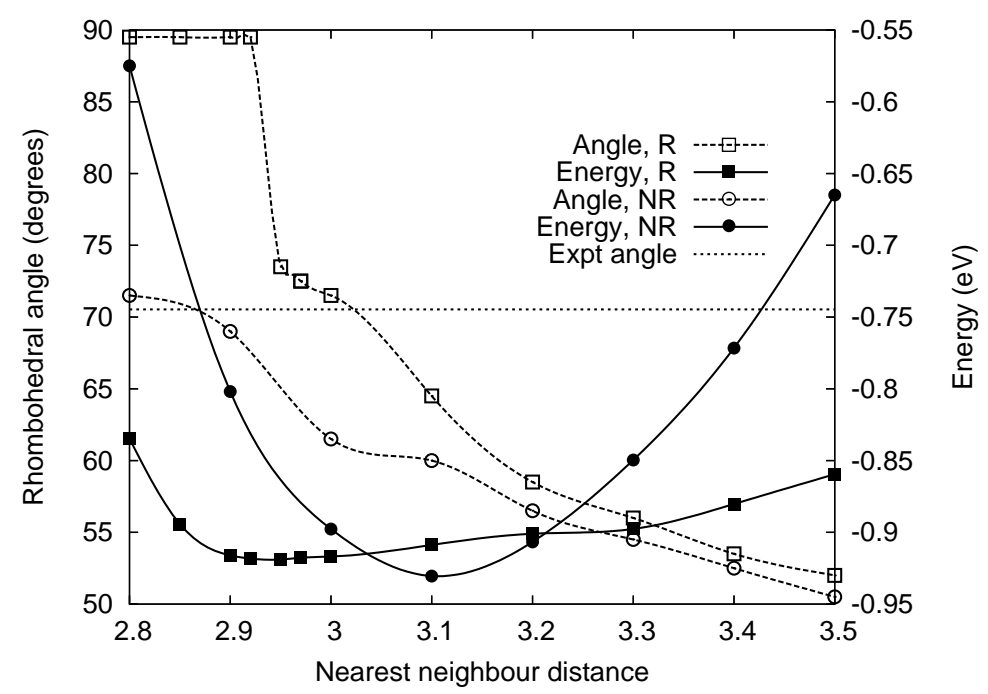

FIG. 4: The effect of relativity on the stability of the rhombohedral lattice in mercury. At each bond length, the optimized energy and corresponding angle is plotted. The relativistic (R) and non-relativistic (NR) values are given, with the experimental rhombohedral angle marked by a dotted line. Energy is in eV and nearest neighbor distance is in $\AA$.

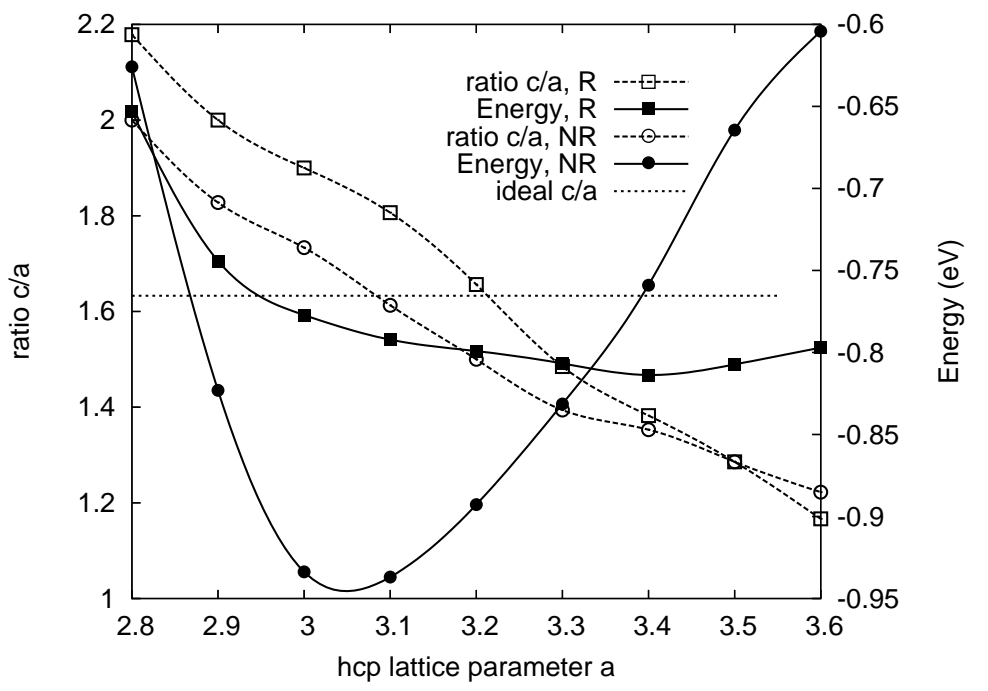

FIG. 5: The effect of relativity on the stability of the hexagonal lattice in mercury. At each value of $a$, the optimized energy and corresponding ratio of $c / a$ is plotted. For mercury, the lattice parameter $a$ is only the nearest neighbor distance for values of $c / a>1.63$. The relativistic (R) and non-relativistic (NR) values are given, with the ideal $c / a$ ratio marked by a dotted line. Energy is in $\mathrm{eV}$ and lattice parameter $a$ is in $\AA$. 


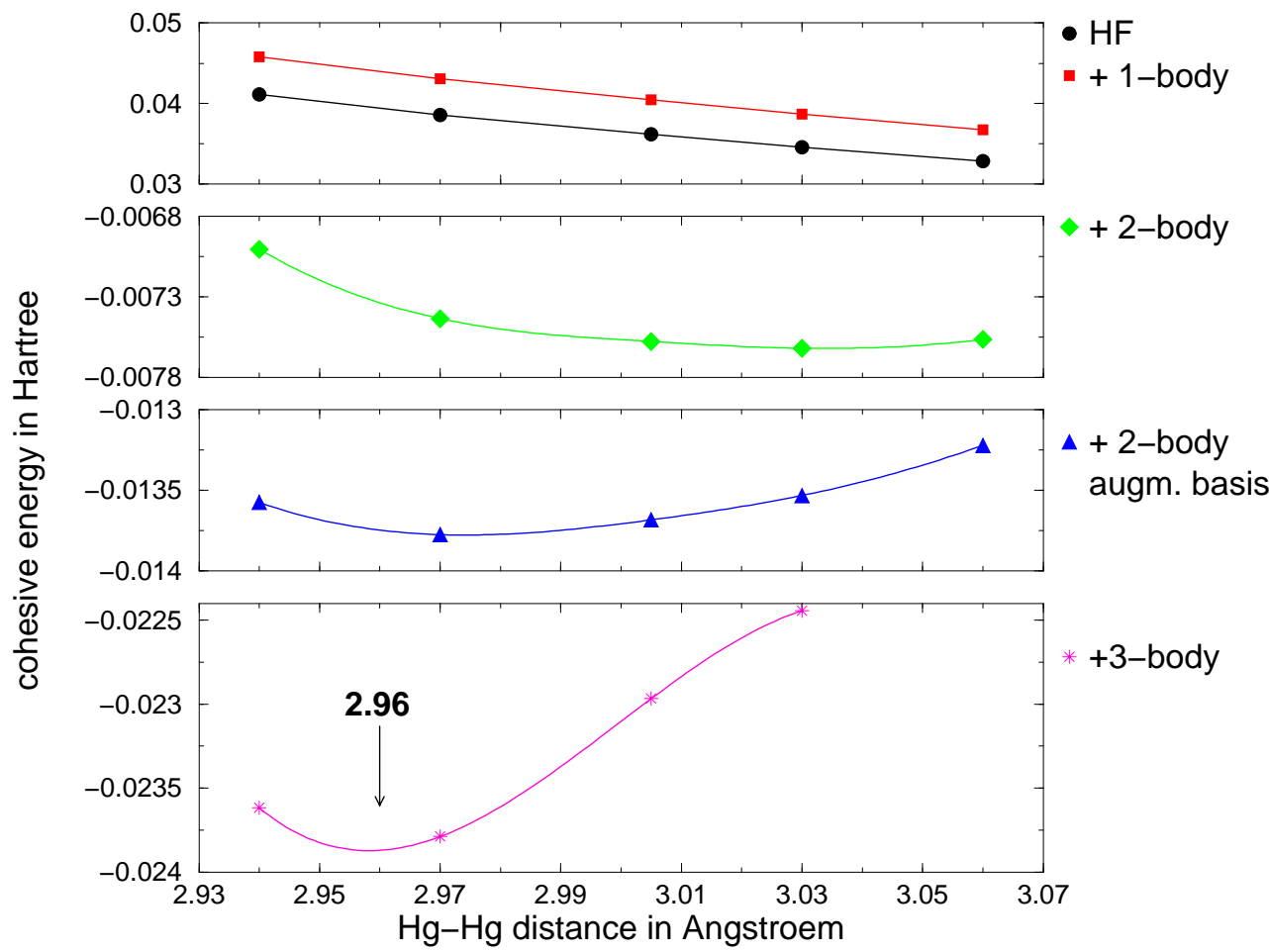

FIG. 6: The cohesive energy of $\mathrm{Hg}$ with respect to the nearest neighbor-distance $a$. 


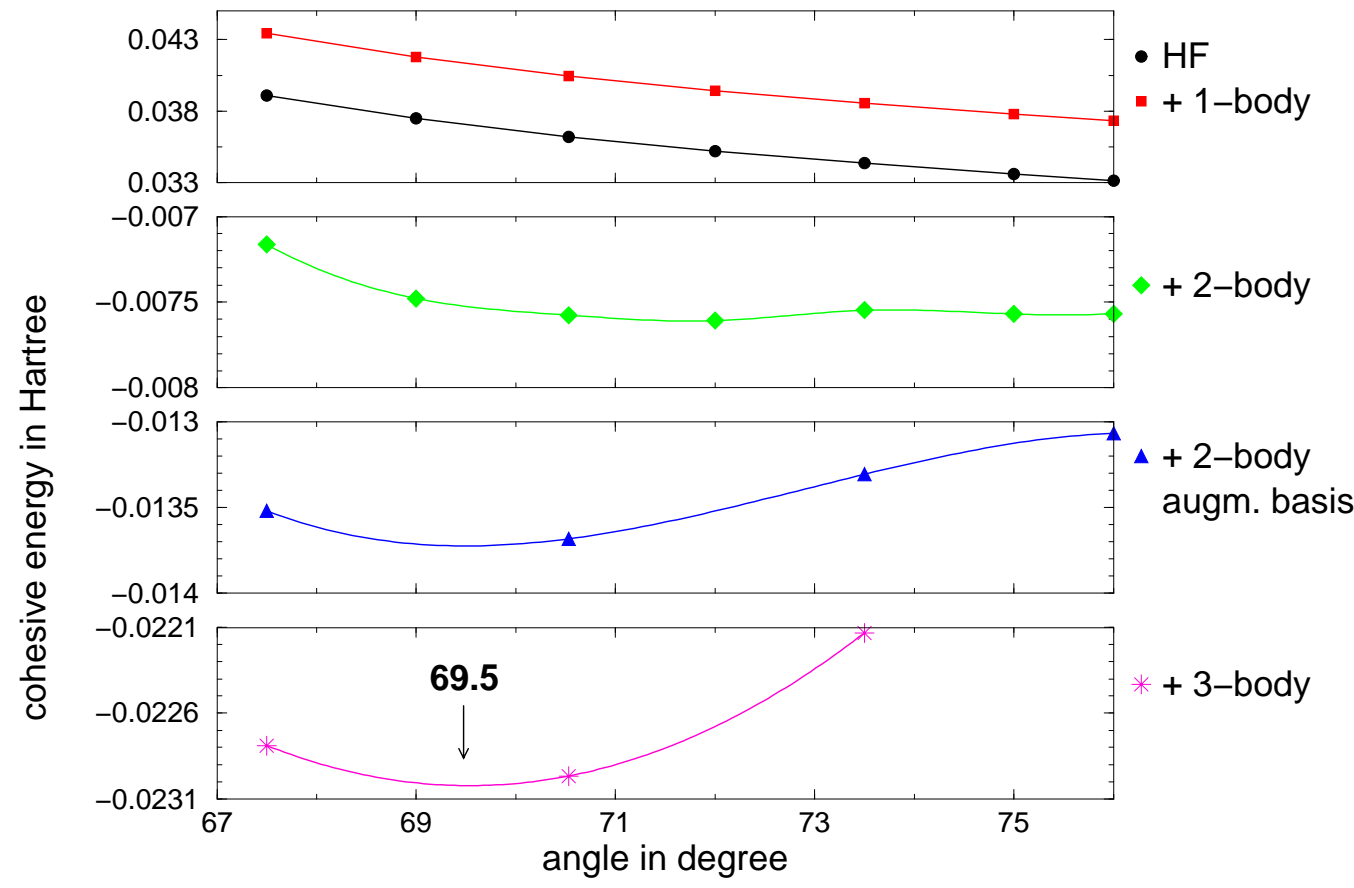

FIG. 7: The cohesive energy of $\mathrm{Hg}$ with respect to the angle in the rhombohedral structure. 

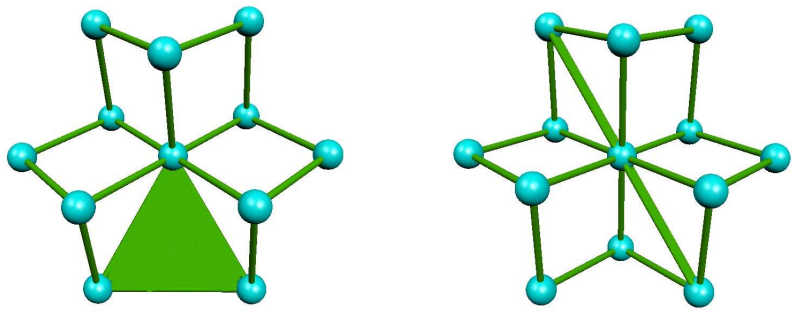

b)

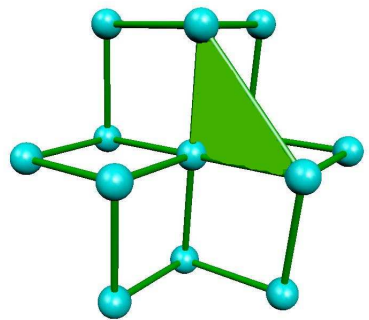

a)

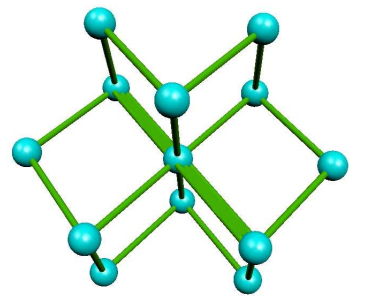

c)

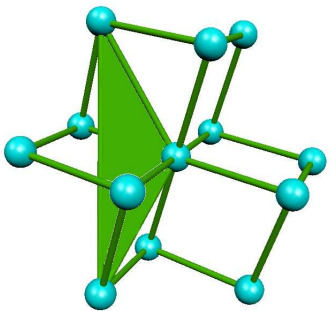

e)

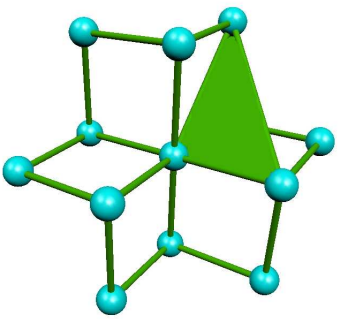

d)

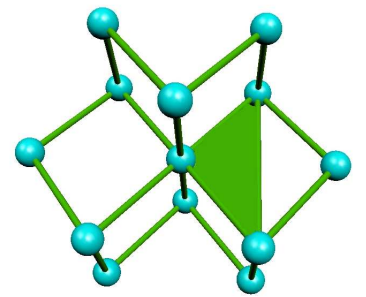

f)

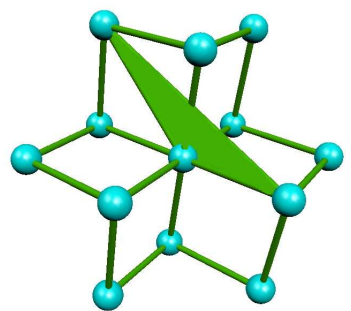

h)

FIG. 8: 3-body clusters considered in the incremental scheme, drawn on a background of the 12 nearest neighbors with all bonds of length $\mathrm{a}_{0}$ drawn. 


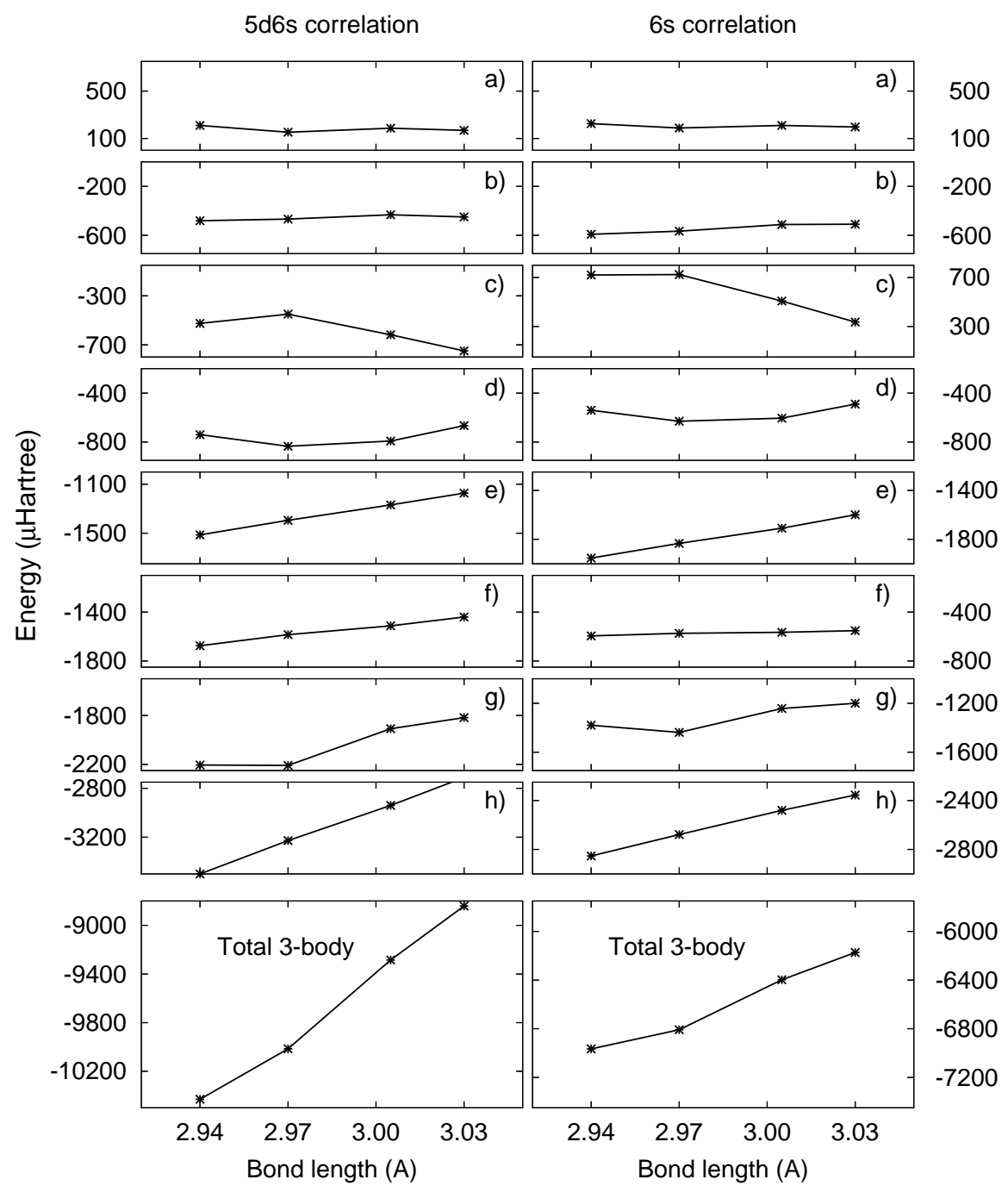

FIG. 9: 3-body results for the nearest neighbor distance of solid mercury. Left: results of the $(5 d 6 s)$ correlation treatment; Right: $6 s$ correlation only. The energies are weighted for the total contribution to the lattice of each increment. The scale is the same in each sub-plot. 


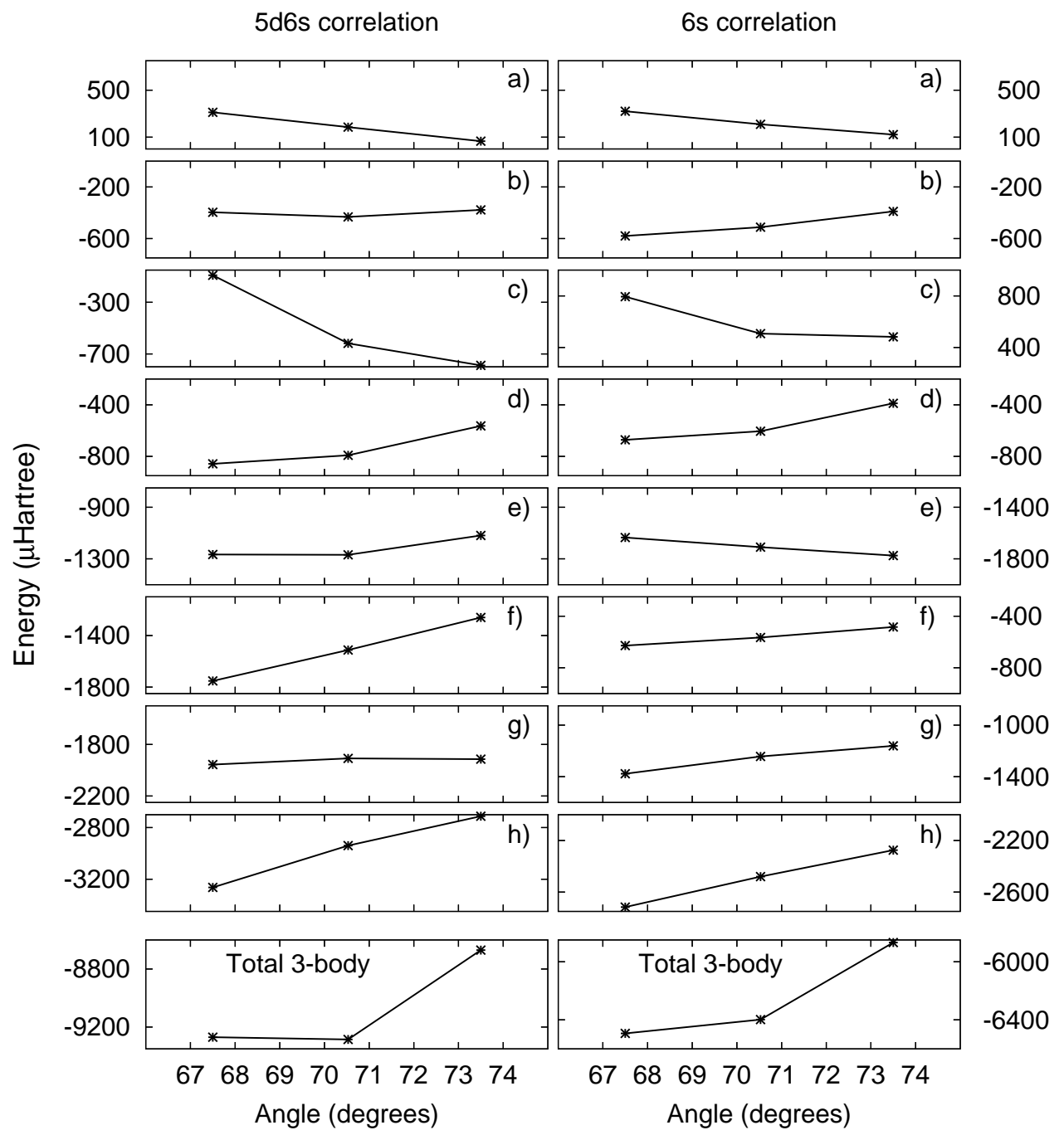

FIG. 10: 3-body results for the optimized angle of solid mercury. Left: results of the (5d6s) correlation treatment; Right: $6 s$ correlation only. The energies are weighted for the total contribution to the lattice of each increment. The scale is the same in each sub-plot. 